\title{
Kinetics of protective antibodies are determined by the viral surface antigen
}

\author{
Daniel D. Pinschewer, ${ }^{1}$ Mar Perez, ${ }^{2}$ Eswaraka Jeetendra, ${ }^{3}$ Thomas Bächi, ${ }^{4}$ Edit Horvath, ${ }^{1}$ \\ Hans Hengartner, ${ }^{1}$ Michael A. Whitt, ${ }^{3}$ Juan Carlos de la Torre, ${ }^{2}$ and Rolf $M$. Zinkernagel ${ }^{1}$
}

\begin{abstract}
1Institute of Experimental Immunology, Department of Pathology, University Hospital of Zurich, Zurich, Switzerland. 2Department of Neuropharmacology, The Scripps Research Institute, La Jolla, California, USA. ${ }^{3}$ Department of Molecular Sciences, University of Tennessee Health Science Center (UTHSC), Memphis, Tennessee, USA. ${ }^{4}$ Laboratory for Electron Microscopy, University of Zurich, Zurich, Switzerland.
\end{abstract}

\begin{abstract}
Delayed and weak virus neutralizing antibody $(\mathrm{nAb})$ responses represent a hallmark correlating not only with the establishment of persistent infection but also with unsuccessful vaccine development. Using a reverse genetic approach, we evaluated possible underlying mechanisms in 2 widely studied viral infection models. Swapping the glycoproteins (GPs) of lymphocytic choriomeningitis virus (LCMV, naturally persisting, noncytolytic, inefficient $\mathrm{nAb}$ inducer) and vesicular stomatitis virus (VSV, nonpersisting, cytolytic, potent $\mathrm{nAb}$ inducer) transferred the only target of nAb's from either virus to the other. We analyzed the nAb response to each of the 2 recombinant and parent viruses in infected mice and found that $\mathrm{nAb}$ kinetics were solely determined by the viral surface GP and not by the virus backbone. Moreover, the slowly and poorly nAb-triggering LCMV virion was a potent immunogenic matrix for the more antigenic VSV-GP. These findings indicate that the viral GP determines nAb kinetics largely independently of the specific viral infection context. They further suggest that structural features of viral GPs or coevolutionary adaptation of the virus's GP to the host's naive $B$ cell repertoire, or both, may critically limit $\mathrm{nAb}$ kinetics and improvement of vaccine efficacy.
\end{abstract}

\section{Introduction}

Induction of neutralizing antibodies (nAb's) represents a central protective mechanism of most currently available antiviral vaccines (1). Acute cytolytic virus infections typically elicit quickly (i.e., within $4-8$ days) a potent and protective $\mathrm{nAb}$ response. In contrast, attempts to induce protective antibody immunity against persisting viruses such as HIV have repeatedly failed despite considerable efforts in the face of worldwide HIV/AIDS pandemics (2). Similar difficulties are encountered with hepatitis $C$ virus (3), an important cause of end-stage liver disease. These viruses induce typically delayed and comparably weak $\mathrm{nAb}$ responses in infected individuals. Several mechanisms - not mutually exclusive - have been proposed. Structural features of the HIV glycoprotein (GP) render antibody-mediated neutralization difficult $(4,5)$. Repeated $\mathrm{nAb}$ escape through high GP sequence variation in the rapidly growing viral quasispecies contributes further to inefficient antibody-mediated virus control $(6,7)$. Additionally, T cell-mediated immunopathologic or suppressive effects on antibody production have been proposed (8-13).

Here we examined the individual impact of the viral GP, the target of nAb's, versus the viral backbone in determining either rapid or delayed nAb kinetics. Using a reverse genetic approach we swapped

Nonstandard abbreviations used: ARM, Armstrong ARM53b; GP, glycoprotein; HIS, hyperimmune serum; i.p., intraperitoneal; IND, VSV serotype Indiana; INDG, IND GP; LCMV, lymphocytic choriomeningitis virus; 2-ME, 2-mercaptoethanol; nAb, neutralizing antibody; NJG, VSV serotype New Jersey GP; NP, nucleoprotein; rLCMV, recombinant LCMV; rLCMV/INDG, rLCMV expressing INDG; rLCMV/NJG, rLCMV expressing NJG; RT, room temperature; rVSV, recombinant VSV; rVSV/LCMV-GP, rVSV expressing LCMV-GP; UV, ultraviolet light; VSV, vesicular stomatitis virus.

Conflict of interest: M.A. Whitt is currently an employee of GTx Inc., which has licensed a patent from the University of Tennessee Research Foundation on the use of recombinant VSV as an oncolytic vector. The work described in this article was completed at UTHSC before M.A. Whitt's employment with GTx Inc.

Citation for this article: J. Clin. Invest. 114:988-993 (2004).

doi:10.1172/JCI200422374 the GPs of the prototypic arenavirus lymphocytic choriomeningitis virus (LCMV; Figure 1, A-C) and of the prototypic rhabdovirus vesicular stomatitis virus (VSV; Figure 1, A-C). LCMV is a natural mouse pathogen found in wildlife as persistent, noncytolytic and vertically transmitted infection $(14,15)$. Acute infection of adult mice is controlled by a vigorous CTL response. Virus-neutralizing serum activity remains either undetectable or is found at low titers and with a delay of greater than $60-100$ days $(10,14,15)$. LCMV $n A b$ 's are directed against epitopes in the GP-1 portion of the virus GP. Experimental infection of mice with the highly cytolytic VSV evokes an early (4 days or less) and potent GP-specific nAb response (16) that prevents lethal myeloencephalitis (17). The recombinant LCMV (rLCMV) expresses the VSV serotype Indiana (IND) GP (INDG) instead of its own GP (Figure 1, A-C) (18). It is efficiently neutralized by VSV nAb's in vitro but has retained the noncytolytic behavior of LCMV in persistently infected mice. For the present study we generated the reverse combination, a recombinant VSV (rVSV) expressing LCMV-GP (rVSV/LCMV-GP) instead of its own GP (Figure 1, A-C). Infection of mice with either of the two recombinant or WT viruses revealed a critical role for the GP but not for the viral backbone in determining $n A b$ kinetics.

\section{Results}

At first, we studied the particle morphology and surface determinants of rLCMV expressing INDG (rLCMV/INDG) and of rVSV/LCMV-GP by electron microscopy of infected cells. Budding rLCMV/INDG and LCMV-Armstrong ARM53b (LCMVARM) particles were both pleomorphic, of variable diameter, and exhibited the arenavirus typical "sandy" interior due to incorporated ribosomes (15) (Figure 1C). Their surface reacted with either INDG- or LCMV-GP-specific antibodies and immunogold, as predicted (Figure 1C). The rVSV/LCMV-GP showed the bullet-shaped particle morphology of its parent VSV-IND, exhibiting, however, exclusively LCMV-GP on its surface (Figure 1C). We have previous- 
A LCMV-ARM

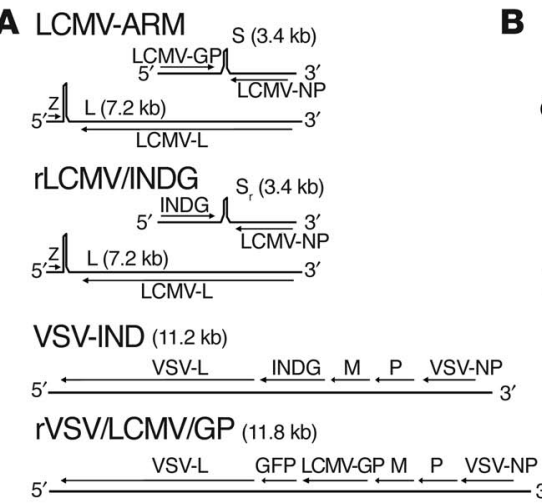

C

C LCMV-ARM

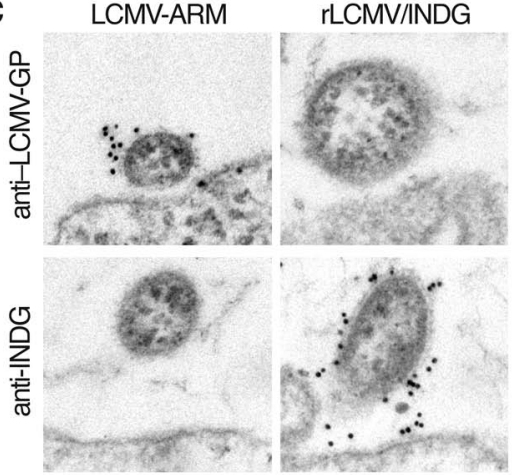

D

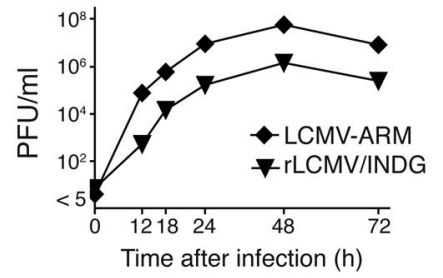

B

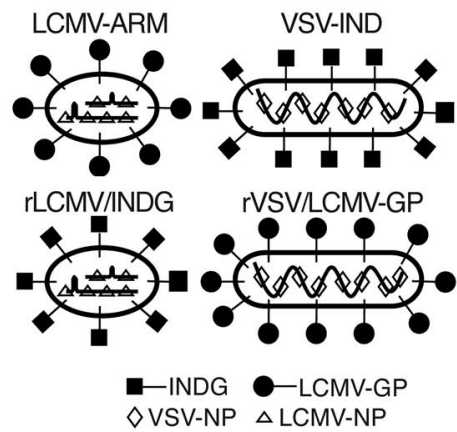

IVSVILCMV-GP VSV-IND
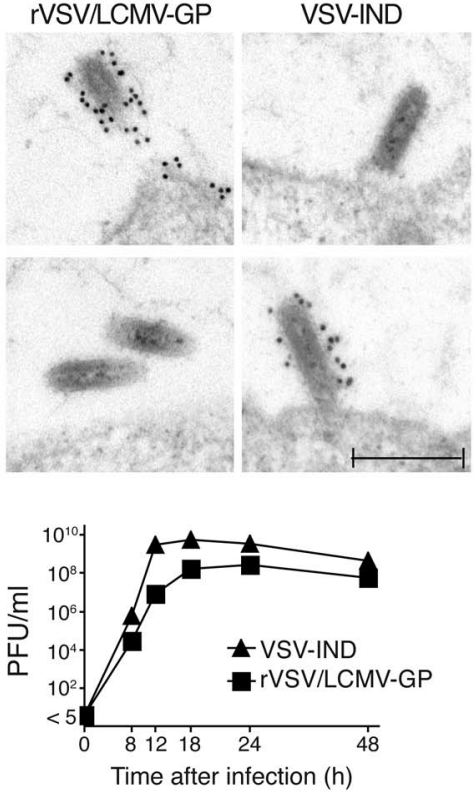

Figure 1

WT and recombinant viral genomes. (A) The LCMVARM genome consists of 2 single-stranded negativestrand ambisense RNA segments. The long $(L)$ segment encodes for the LCMV RNA-dependent RNA polymerase $(R d R p) L$ and for the matrix protein $Z$, while the short (S) segment carries the GP and NP genes. In rLCMV/INDG the LCMV-GP gene of LCMV-ARM is substituted for INDG (18). The single-stranded negativestrand RNA genome of VSV-IND encodes in $3^{\prime}-5^{\prime}$ orientation for NP, phosphoprotein $(P)$, matrix protein $(M)$, INDG, and $L$. $L$ and $P$ are associated in the VSV RdRp complex (16). rVSV/LCMV-GP expresses GFP from an additional transcription unit between $L C M V-G P$ and $L$. (B) Schematic describing the topology of GPs and NPs of the above viruses on the surface and inside infectious particles, respectively. (C) Immunogold staining of viral particles budding from BHK-21 cells infected with the viruses as indicated. The nAb's of the indicated specificity were used for detection of the viral surface antigens. Scale bar: $0.2 \mu \mathrm{m}$. (D) BHK-21 cells were infected with the indicated viruses at a $\mathrm{MOI}$ of 0.01 . Infectious virus in the supernatant was measured at the indicated time points. Symbols represent the mean of quadruplicate samples (SD within the size of the symbols). ly reported that propagation and peak titers of rLCMV/INDG are reduced when compared with LCMV-ARM (18). In vitro growth curves revealed that rVSV/LCMV-GP was similarly attenuated when compared with VSV-IND (Figure 1D). We next compared the kinetics of the nAb response elicited by rLCMV/INDG, rVSV/ LCMV-GP, and the two parent viruses LCMV and VSV (Figure 2). We infected C57BL/ 6 mice with $2 \times 10^{4}$ PFU LCMV-ARM, rLCMV/ INDG, VSV-IND or rVSV/LCMV-GP, or with $2 \times 10^{7} \mathrm{PFU} \mathrm{rVSV/}$ LCMV-GP. Serum samples were collected at various time points to test for protective virus nAb's in neutralization assays and for nonprotective antibodies binding to the virus's internal nucleoproteins (NPs) in an ELISA (Figure 2). Unlike the typically late LCMV-specific $\mathrm{nAb}$ response, $\mathrm{rLCMV}$ /INDG evoked VSV nAb's within 2-3 days after infection (Figure 2A, Figure 3C) with an isotype class switch to neutralizing IgG around day 6-8 (Figure 2A and data not shown). VSV nAb kinetics in VSV-infected mice followed a similar pattern (Figure 2A). Notably, though, several independent experiments showed that rLCMV/INDG elicited a neutralizing IgM and IgG response that was either faster or equally fast as VSV. Neither LCMV nor rVSV/LCMV-GP induced detectable VSV-IND nAb's because they lacked INDG. All VSV-neutralizing serum samples neutralized rLCMV/INDG in vitro (Figure 2C), confirming that rLCMV/INDG infectivity was INDG-mediated (18). But neither of the sera collected during this experiment exhibited neutralizing activity against rVSV/LCMV-GP (Figure 2B) or LCMV-ARM (Figure 2D). Both viruses were, however, neutralized by LCMV-GP1specific neutralizing mAb WEN-3 and by LCMV-ARM hyperimmune serum (HIS), demonstrating that rVSV/LCMV-GP infectivity was mediated by correctly folded LCMV-GP on its surface. One explanation for the failure of rVSV/LCMV-GP to induce LCMVGP-specific nAb's could have been that antigen expression in vivo was reduced as compared with VSV-IND. We used VSV-NP-specific (also known as VSV N-specific) IgG responses as a surrogate marker for the total amount of viral antigen expressed in infected animals over time. In a validation experiment, VSV-NP-specific IgG was not detected after immunization with up to $2 \times 10^{5} \mathrm{PFU}$ equivalents of ultraviolet light-inactivated (UV-inactivated) VSVIND (not shown). As predicted, mice infected with LCMV-ARM or with rLCMV/INDG lacking VSV-NP (Figure 1) produced no VSVNP-specific IgG (Figure 2E). Compared with VSV-IND-immunized animals, rVSV/LCMV-GP-infected mice mounted equal or even slightly higher VSV-NP-specific IgG titers when given the same dose (10 $10^{\mathrm{PFU}}$ ) or $2 \times 10^{7} \mathrm{PFU}$ rVSV/LCMV-GP, respectively (Figure $2 \mathrm{E}$ and data not shown). Hence, the failure of rVSV/LCMV-GP to induce nAb's was unlikely to be due to insufficient in vivo antigen expression. As expected, LCMV-NP-binding IgG was only 

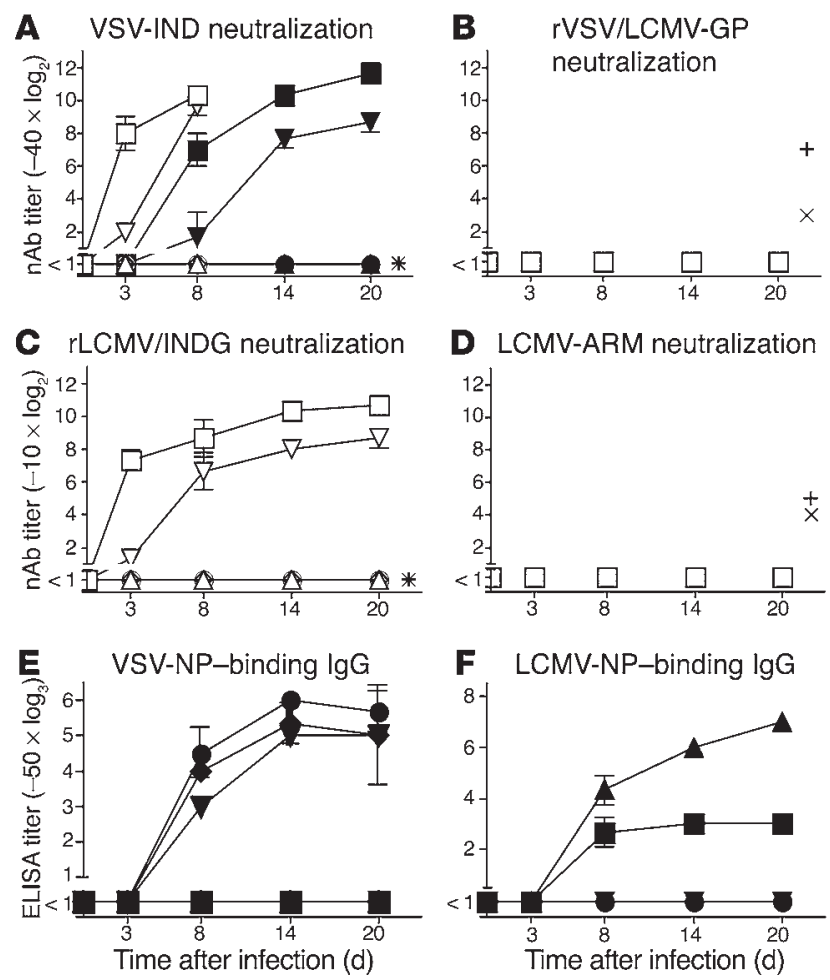

detectable in LCMV-ARM and rLCMV/INDG immune sera, and titers were higher in the former than the latter (Figure $2 \mathrm{~F}$ ), which was probably due to reduced propagation kinetics of rLCMV/ INDG (18) resulting in reduced in vivo antigen expression.

Rapid nAb kinetics in $\mathrm{rLCMV} / \mathrm{INDG}$ infection were surprising considering the poor $\mathrm{nAb}$ induction by the parent virus LCMV. We therefore studied dose response, $\mathrm{CD}^{+} \mathrm{T}$ cell dependence, and protective capacity of rLCMV/INDG-induced nAb's. We inoculated C57BL/ 6 mice i.v. with titrated doses of live rLCMV/INDG ranging from 0.1 to $10^{4} \mathrm{PFU}$ or with $10^{4} \mathrm{PFU}$ equivalents of UV-inactivated rLCMV/INDG. The dose of live rLCMV/INDG influenced peak titers and initial kinetics of the VSV-nAb's response (Figure 3, $A$ and $B$ ). UV inactivation reduced the capacity of rLCMV/INDG to induce total VSV-neutralizing Ig by a factor of approximately 1,000 , and nAb's titers were short lived. Low doses (0.1-10 PFU) of live rLCMV/INDG (Figure $3 \mathrm{~B}$ and data not shown) but not the UV-inactivated equivalent of $10^{4} \mathrm{PFU}$ induced neutralizing $\mathrm{IgG}$, indicating that the $\mathrm{nAb}$ response to $\mathrm{rLCMV} / \mathrm{INDG}$ resulted

\section{Figure 3}

Virus dose response and CD4+ $\mathrm{T}$ cell dependence of the $\mathrm{rLCMV} /$ INDG-induced $\mathrm{nAb}$ response. (A and B) C57BL/6 mice were infected with 0.1 PFU (asterisks), 1 PFU (filled squares), 10 PFU (filled triangles), $10^{2}$ PFU (filled inverted triangles), $10^{3}$ PFU (filled diamonds), $10^{4}$ PFU (filled circles) live or $10^{4}$ PFU equivalents of UV-inactivated (open circles) rLCMV/INDG i.v. Serum samples collected at the indicated time points were tested for VSV-neutralizing total $\lg (\mathbf{A})$ and $\lg G(B)$. (C) C57BL/6 mice depleted of CD4 ${ }^{+} \mathrm{T}$ cells (open symbols) or undepleted (filled symbols) were infected with rLCMV/INDG i.v. on day 0. Serum samples collected at the indicated time points were tested for VSV-neutralizing total Ig (squares) and IgG (triangles). Symbols represent the mean of 2 ( 0.1 PFU group in $\mathbf{A}$ and $\mathbf{B}$ ) to 3 mice (remainder groups and panels) per group $\pm \mathrm{SD}$. One representative experiment of 2 similar experiments is shown.

\section{Figure 2}

Correlation of $\mathrm{nAb}$ induction with the viral surface determinant. C57BL/6 mice were infected i.v. with $2 \times 10^{4} \mathrm{PFU}$ rLCMV/INDG (squares), LCMV-ARM (triangles), VSV-IND (inverted triangles), rVSV/ LCMV-GP (diamonds), or with $10^{7}$ PFU rVSV/LCMV-GP (circles). Serum samples collected at the indicated time points were tested in neutralization assays (A-D) or by ELISA ( $E$ and $\mathbf{F}$ ) for total Ig (open symbols) and IgG (filled symbols) with the following specificities: (A) VSV-IND-neutralizing total Ig and -neutralizing IgG; (B) rVSV/LCMVGP-neutralizing total Ig; (C) rLCMV/INDG-neutralizing total Ig; (D) LCMV-ARM-neutralizing total Ig; (E) VSV-NP-binding IgG; (F) LCMVNP-binding IgG. Except for ARM-HIS $(x)$ and WEN-3 mAb (+) controls (single samples), symbols generally represent the mean of 3 mice per group \pm SD. Due to limitations in the amount of serum collected, for some groups and time points in D-F only 2 out of 3 mice could be tested. One representative of 2 experiments is shown.

largely from productive infection. Even in $\mathrm{CD} 4^{+} \mathrm{T}$ cell-depleted mice, rLCMV/INDG induced a short-lived VSV-nAb response (presumably IgM) that was largely independent of cognate $\mathrm{T}$ cell help, while isotype class switch to IgG was abrogated (Figure 3C). This underlined the extraordinary immunogenicity that the LCM virion provided for the foreign INDG surface determinant but not for its own LCMV-GP. The protective capacity of rLCMV/INDGinduced nAb's was demonstrated in highly VSV-susceptible type I IFN receptor-deficient mice ( $A 129$; $\mathrm{LD}_{50}$ less than 50 PFU i.v.) (19). Immunization with rLCMV/INDG, but not with rLCMV expressing VSV serotype New Jersey GP (rLCMV/NJG; D.D. Pinschewer, unpublished data), induced VSV-IND-neutralizing IgG (Figure 4A) and protected $A 129$ mice from $5 \times 10^{6} \mathrm{PFU}$ VSV-IND i.v. (more than $1,000 \times$ LD $_{50}$; Figure 4B).

We observed that in comparison to LCMV-ARM, rLCMV/INDG induced CTLs of comparable frequency as tested by MHC class I tetramer staining, but these cells exhibited reduced cytolytic effector function (Supplemental Figure 1; supplemental material available at http://www.jci.org/cgi/content/full/114/7/988/DC1). This was apparently not due to nAb-mediated premature elimination of rLCMV/INDG (Supplemental Figure 1) and raised the concern that LCMV-ARM infection may exhibit dominant negative effects on $\mathrm{nAb}$ responses $(8-13)$. Such effects could have been mediated by $\mathrm{T}$ cells or other unknown factors and could have been missed in the experiments discussed above. To address this possibility
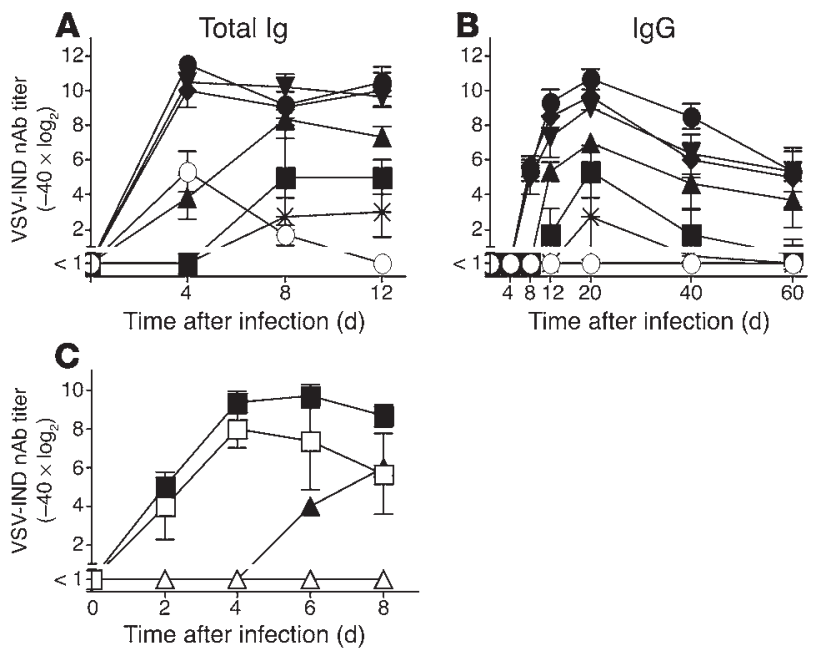


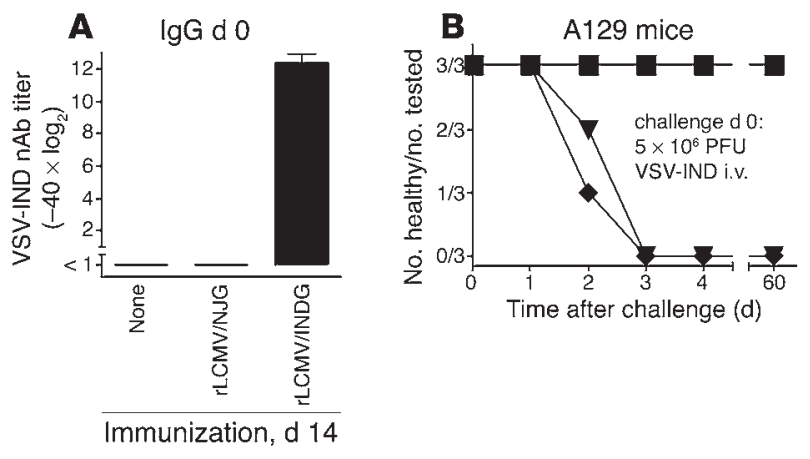

\section{Figure 4}

Protective capacity of the $\mathrm{rLCMV/INDG-induced} \mathrm{nAb} \mathrm{response.} \mathrm{A129}$ mice were immunized on day 14 prior to challenge with live rLCMV/ INDG (right bar in $\mathbf{A}$, squares in $\mathbf{B}$ ) or rLCMV/NJG (center bar in $\mathbf{A}$, inverted triangles in $\mathbf{B}$ ), or were left uninfected (left bar in $\mathbf{A}$, diamonds in B). (A) On day 0, serum samples were tested for VSV-IND-neutralizing IgG, and all mice were challenged i.v. with $5 \times 10^{6}$ PFU VSV-IND. (B) Daily monitoring for clinical signs of terminal myeloencephalitis. Symbols represent the mean of 3 mice per group \pm SD. One representative experiment of 2 similar experiments is shown.

and to further rule out potential nonspecific immunostimulatory effects of rLCMV/INDG on nAb responses, we infected C57BL/6 mice i.v. with $2 \times 10^{4} \mathrm{PFU}$ of either rLCMV/INDG or LCMV-ARM alone or with a combination of both (Figure 5). Such coinfection resulted in as vigorous a CTL response as that which occurs after LCMV-ARM infection alone (Supplemental Figure 2) but neither suppressed VSV-neutralizing total Ig production (Figure 5A) nor accelerated LCMV-ARM-specific nAb responses (Figure 5C). As demonstrated by adoptive transfer of LCMV-ARM-immune splenocytes, the slight reduction in VSV-IND neutralizing IgG (Figure 5B) in rLCMV/INDG plus LCMV-ARM-coinfected animals was not due to direct suppressive effects of LCMV immune $T$ cells (Supplemental Figure 3). It was therefore more likely to be the result of LCMV-induced immunopathology disturbing lymphoid microarchitecture and compromising B cell/T cell cooperation and isotype class switch (20).

\section{Discussion}

The present data show that the kinetics of $\mathrm{nAb}$ responses are largely intrinsic to the viral target of nAb's, for example, the GPs of LCMV and VSV in our study. They further indicate that immunogenic properties of the viral backbone seem unlikely to represent a main limiting factor for the host's $\mathrm{nAb}$ response. As in human HIV infection $(4,6)$, the genetic variability of LCMV (21) can contribute greatly to the lack of nAb-mediated virus con-

\section{Figure 5}

Resistance of the rLCMV/INDG-induced $n A b$ response to LCMVARM coinfection. C57BL/6 mice were infected i.v. with $2 \times 10^{4} \mathrm{PFU}$ LCMV-ARM (triangles), $2 \times 10^{4} \mathrm{PFU}$ rLCMV/INDG (squares), or with both above inocula (circles). Serum samples collected at the indicated time points were tested for VSV-IND-neutralizing total Ig (A) and IgG (B) and for LCMV-ARM nAb's (C). ARM-HIS ( $x$ ) and WEN-3 mAb (+) served as controls (single samples). The remaining symbols generally represent the mean of three mice per group $\pm S D$. Due to limitations in the amount of serum collected, for some groups and time points in $\mathbf{C}$ only 2 out of 3 mice could be tested. One representative experiment of 2 similar experiments is shown. trol. It remains, however, to be determined whether viral immune escape from $\mathrm{nAb}$ could be limited if new viral variants elicited a $\mathrm{nAb}$ response within days rather than months after their emergence. In a further analogy to HIV, LCMV has evolved "glycan shields" $(4,22)$ and possibly also a GP-conformational epitope masking (5) as molecular mechanisms to avoid efficient antibody-mediated neutralization. By an alternative and not mutually exclusive mechanism, viral GPs may have coevolved to fit the naive antibody repertoire of the natural host only marginally (23); that is, B cells with a receptor of neutralizing specificity may exist at very low frequencies or may only be generated by substantial somatic hypermutation. Comparably more potent LCMV nAb responses in non-natural hosts such as guinea pigs, humans, or mice genetically engineered to express a LCMV-neutralizing B cell receptor $(11,12)$ argue in favor of such a mechanism.

Further studies in other viral systems will be necessary to evaluate whether our findings and conclusions can be generalized to other virus-host combinations and in particular to clinically relevant infections such as HIV or hepatitis C virus. Our data suggest, however, that a viral GP inducing only delayed and low nAb titers in the natural context (e.g., LCMV-GP or HIV gp120) would be unlikely to be a better inducer when provided in another configuration, including an optimal carrier matrix for $\mathrm{nAb}$ induction such as VSV (24). In line with this view, large-scale phase III human trials with recombinant HIV gp120 protein have failed to induce nAbmediated protection against HIV (25), and expression of HIV-GP on a recombinant VSV could not elicit a rapid and strong HIV nAb response in mice (26) or rhesus macaques (27). In these earlier studies the reverse recombinant (i.e., HIV-expressing INDG) was not tested, and therefore these studies could not provide a mechanistic understanding of the underlying problem by mapping it to either the target molecule or to the virion matrix. Notably, LCMV particles display their surface GP at high density and in a highly repetitive, paracristalline array $(28,29)$, just like VSV-IND (16). This pattern has been suggested to represent an optimal trigger for B cells (24). Both recombinant viruses (rLCMV/INDG and rVSV/LCMVGP) contain putatively lower densities of their respective GPs due to the lack of a specific incorporation signal $(15,16)$. Nevertheless, rLCMV/INDG was an optimal inducer of INDG-specific nAb's while the highly repetitive LCMV particle failed to elicit a neutralizing response against LCMV-GP. For haptens, 15 identical epitopes
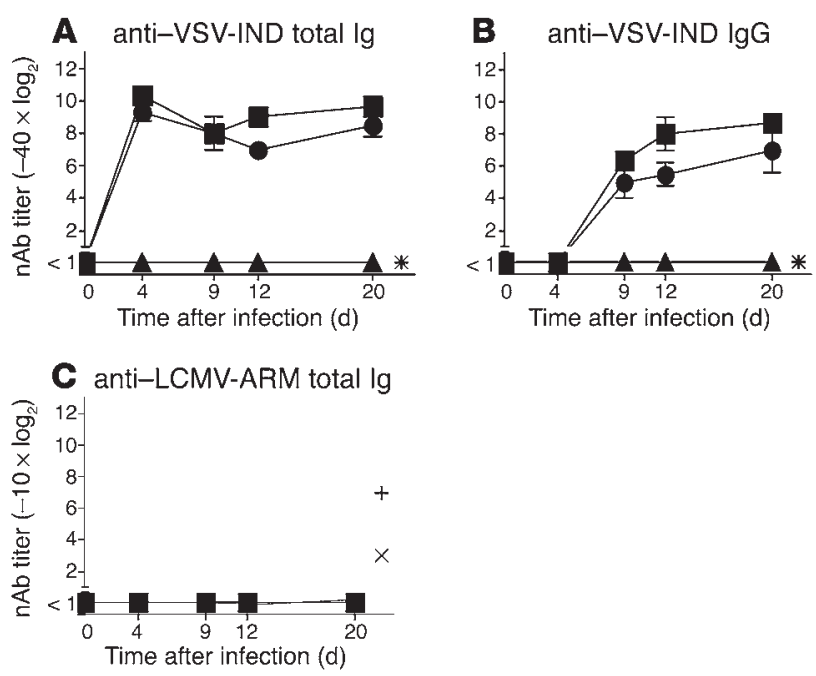
on a linear polymer represent the threshold to induce antibodies in the absence of T cell help (30), while antibody responses to protein antigens are not normally subject to this limitation. The density of immunogold labeling on rVSV/LCMV-GP (Figure 1C) suggested that, like in $\operatorname{LCMV}(28,29)$, this very stringent criterion had been met but the virus failed to induce a $\mathrm{nAb}$ response. Hence, the viral surface antigen sets clear limits to nAb kinetics, apparently independently of its pattern on viral particles. Within the range of this limitation, vector properties allowing for delivery of high antigen doses (31) and optimal B cell triggering (24) will certainly contribute to optimize vaccine responses.

Taken together, the present finding that the murine (the natural host) LCMV infection is optimally immunogenic for the heterologous INDG protein but almost unable to elicit a protective $\mathrm{nAb}$ response to LCMV-GP suggests that even the most immunogenic vaccine carrier matrix may in many instances fail to induce rapid and potent (i.e., escape preventing) nAb-mediated immunity against persistent viral diseases.

\section{Methods}

Viruses, virus titration, and UV inactivation. LCMV-ARM is a triple plaque-purified isolate of ARM CA 1371 (32) obtained originally from M.J. Buchmeier (The Scripps Research Institute, La Jolla, California, USA). Generation of rLCMV/INDG has been described (18). VSV-IND was originally obtained from D. Kolakofsky (University of Geneva, Geneva, Switzerland). To generate rVSV/LCMV-GP, the LCMV-GP ORF was amplified from PC-LCMV-GP (18) with primers 5'-CGACGCGTTGACACTATGGGTCAGATTGTGACAATG$3^{\prime}$ and $5^{\prime}$-CTAGCTAGCTCAGCGTCTTTTCCAGACGGTTTTTACACC-3' and was cloned into the MluI and NheI sites (corresponding sites in the primers in italics) of the VSV $\Delta$ G-PL/GFP vector (33). The infectious virus was rescued following established protocols (33). The LCMV-GP gene in rVSV/LCMV-GP was sequenced and exhibited a single amino acid change at position 197 (threonin to alanine) as compared with the sequence in pC-LCMV-GP. The latter was found to be identical to the that recently published (34). All viruses were propagated on BHK-21 cells. UV inactivation of rLCMV/INDG and VSV was done as previously described (35). A Vero cell plaque assay was used to quantify VSV-IND and rVSV/LCMV-GP infectivity (36). LCMV and rLCMV/INDG were titrated by an immunofocus assay (37). Generation and handling of recombinant viruses under BSL-2 conditions was approved by the Institutional Biosafety Committee of The Scripps Research Institute (La Jolla, California, USA) and by the Recombinant DNA Committee of the University of Tennessee (Memphis, Tennessee, USA), respectively. In compliance with Swiss federal law we made a report to and a corresponding registration with the Swiss Agency for the Environment, Forests, and Landscape (Bern, Switzerland).

Mice and animal experiments. C57BL/ 6 were obtained from the Institut für Labortierkunde, University of Zurich. Animal experiments including infection with recombinant viruses were carried out with authorization of the Veterinäramt of the Kanton Zurich and in accordance with the Swiss law for animal protection. All mice exhibiting hind limb paralysis as sign of terminal VSV myeloencephalitis or LCMV-induced choriomeningitis with convulsions were sacrificed in accordance with the aforementioned regulations.

In vivo $C D 4^{+} T$ cell depletion. Mice were depleted of $\mathrm{CD} 4^{+} \mathrm{T}$ cells by two intraperitoneal (i.p.) injections of $1 \mathrm{mg}$ anti-CD4 antibody YTS 191.6 (38) (the hybridoma was a generous gift of $\mathrm{H}$. Waldmann, University of Oxford, Oxford, United Kingdom) on days 3 and 1 prior to infection. The efficiency of depletion (> 99\%) was confirmed by flow cytometry of peripheral blood cells on the day of infection.

Antibodies, detection, and generation of LCMV-ARM byperimmune serum. The nAb's against LCMV and rLCMV/INDG were detected in a focus reduction assay as previously described for LCMV (39). VSV and rVSV/LCMV-GP nAb's were measured by standard plaque reduction assays for VSV (40). VSV-neutralizing IgG was determined after inactivation of IgM by 2-mercaptoethanol (2-ME) (41). Despite controversies over the specificity of this procedure it remains the only method for determining virus neutralizing IgG reliably in a plaque reduction assay because specific secondary antibodies cannot be used. Hence, we refer to untreated nAb activity as total Ig or IgM and to the 2-ME-resistant fraction as neutralizing IgG.

LCMV-NP- and VSV-NP-binding antibodies were detected by ELISA against baculovirus-derived recombinant protein as previously described (31).

For induction of LCMV-ARM HIS, we depleted ten C57BL/6 mice of $\mathrm{CD}^{+} \mathrm{T}$ cells as previously described (21) and infected them with $2 \times 10^{5}$ PFU LCMV-ARM i.p. Four months later, the mice were again inoculated with the same dose of virus via the same route, and another month later they were given in vitro LCMV-ARM-infected primary peritoneal macrophages by i.p. adoptive transfer. Serum samples were collected from all mice during the following weeks and were pooled for the experiments described herein.

The LCMV-GP1-specific neutralizing $\mathrm{mAb}$ WEN-3 has been described previously (42).

$E M$. Monolayers of BHK-21 cells infected with LCMV (48 h, MOI = 0.01), $\mathrm{rLCMV} / \mathrm{INDG}(48 \mathrm{~h}, \mathrm{MOI}=0.1)$, VSV-IND or $\mathrm{rVSV} / \mathrm{LCMV}-\mathrm{GP}(20 \mathrm{~h}$, $\mathrm{MOI}=0.01$ ) were fixed in situ in PBS containing a mixture of $3 \% \mathrm{PBS}$ and $0.1 \%$ glutaraldehyde for $5 \mathrm{~min}$ at room temperature (RT), brought into suspension by scratching, and were further fixed in the same medium for 10 min. After removal of the fixative and washing by centrifugation, the cells were resuspended in PBS and each sample was divided in two and incubated (60 min, RT) with primary mouse anti-INDG mAb VI-7 or mouse anti-LCMV-GP mAb WEN-3, respectively, followed by incubation (60 min, $\mathrm{RT}$ ) with secondary goat anti-mouse IgG conjugated to 10-nm gold particles (Aurion). Excess antibody was washed out and the cells were fixed with $3 \%$ glutaraldehyde $(30 \mathrm{~min}$ ) and $2 \%$ osmium tetroxide (additional 30 $\mathrm{min}$ ), followed by dehydration and thin sectioning in Epon-Araldite. Thin sections were stained with lead citrate and uranyl acetate and examined with a Philips EM 208 transmission electron microscope.

\section{Acknowledgments}

This work was supported by grants of the European Union (Forschungsprogramm LIFE/INFECTIOUS DISEASES, to R.M. Zinkernagel), the Swiss National Science foundation (to D.D. Pinschewer, H. Hengartner, and R.M. Zinkernagel), the NIH (grants GM-53726, to M.A. Whitt, and AI-47140, to J.C. de la Torre), and by the Canton of Zurich. We wish to thank Barbara Knecht and Therese Bruggmann for excellent technical assistance.

Received for publication June 8, 2004, and accepted in revised form July 27, 2004.

Address correspondence to: Daniel D. Pinschewer, Institute of Experimental Immunology, Department of Pathology, University Hospital of Zurich, Schmelzbergstrasse 12, 8091 Zurich, Switzerland. Phone: 41-1-2552989; Fax: 41-1-2554420; E-mail: pinschi@pathol.unizh.ch.

\footnotetext{
1. Zinkernagel, R.M. 2001. Maternal Ab’s, childhood

infections, and autoimmune diseases. N. Engl. J.

Med. 345:1331-1335.
}

2. McMichael, A.J., and Hanke, T. 2003. HIV vaccines
1983-2003. Nat. Med. 9:874-880.

3. Forns, X., Bukh, J., and Purcell, R.H. 2002. The challenge of developing a vaccine against hepatitis C virus. J. Hepatol. 37:684-695.
4. Wei, X., et al. 2003. Ab neutralization and escape by HIV-1. Nature. 422:307-312.

5. Kwong, P.D., et al. 2002. HIV-1 evades Ab-mediated neutralization through conformational masking 
of receptor-binding sites. Nature. 420:678-682.

6. Richman, D.D., Wrin, T., Little, S.J., and Petropoulos, C.J. 2003. Rapid evolution of the neutralizing $\mathrm{Ab}$ response to HIV type 1 infection. Proc. Natl. Acad. Sci. U. S. A. 100:4144-4149.

7. Farci, P., et al. 1994. Prevention of hepatitis C virus infection in chimpanzees after Ab-mediated in vitro neutralization. Proc. Natl. Acad. Sci. U. S. A. 91:7792-7796.

8. Shinohara, N., Watanabe, M., Sachs, D.H., and Hozumi, N. 1988. Killing of antigen-reactive B cells by class II-restricted, soluble antigen-specific CD8+ cytolytic T lymphocytes. Nature. 336:481-484.

9. Barnaba, V., Franco, A., Alberti, A., Benvenuto, R., and Balsano, F. 1990. Selective killing of hepatitis B envelope antigen-specific B cells by class I-restricted, exogenous antigen-specific T lymphocytes. Nature. 345:258-260.

10. Planz, O., Seiler, P., Hengartner, H., and Zinkernagel, R.M. 1996. Specific cytotoxic T cells eliminate $\mathrm{B}$ cells producing virus-neutralizing Ab's [erratum 1996, 384:288]. Nature. 382:726-729.

11. Seiler, P., et al. 1998. Enhanced virus clearance by early inducible lymphocytic choriomeningitis virus-neutralizing Ab's in immunoglobulin-transgenic mice. J. Virol. 72:2253-2258.

12. Hangartner, L., et al. 2003. Antiviral immune responses in gene-targeted mice expressing the immunoglobulin heavy chain of virus-neutralizing Ab's. Proc. Natl. Acad. Sci. U. S. A. 100:12883-12888.

13. Hisatsune, T., Nishijima, K., Kohyama, M., Kato, H., and Kaminogawa, S. 1995. CD8+ T cells specific to the exogenous antigen. Mode of antigen recognition and possible implication in immunosuppression. J. Immunol. 154:88-96.

14. Hotchin, J. 1962. The biology of lymphocytic choriomeningitis infection: virus-induced immune disease. Cold Spr. Harbor. Symp. Quant. Biol. 37:479-499.

15. Buchmeier, M.J., Bowen, M.D., and Peters, C.J. 2001. Arenaviridae: the viruses and their replication. In Fields virology. D.M. Knipe et al., editors. Lippincott Williams \& Wilkins. Philadelphia, Pennsylvania, USA. 1635-1668.

16. Rose, J.K., and Whitt, M.A. 2001. Rhabdoviridae: the viruses and their replication. In Fields virology. D.M. Knipe et al., editors. Lippincott Williams \& Wilkins. Philadelphia, Pennsylvania, USA. 1221-1244.

17. Brundler, M.A., et al. 1996. Immunity to viruses in B cell-deficient mice: influence of Ab's on virus persistence and on T cell memory. Eur. J. Immunol. 26:2257-2262.

18. Pinschewer, D.D., Perez, M., Sanchez, A.B., and de la Torre, J.C. 2003. Recombinant lymphocytic choriomeningitis virus expressing vesicular stomatitis virus glycoprotein. Proc. Natl. Acad. Sci. U. S. A. 100:7895-7900.

19. Muller, U., et al. 1994. Functional role of type I and type II interferons in antiviral defense. Science. 264:1918-1921.

20. Odermatt, B., Eppler, M., Leist, T.P., Hengartner, H., and Zinkernagel, R.M. 1991. Virus-triggered acquired immunodeficiency by cytotoxic T-celldependent destruction of antigen-presenting cells and lymph follicle structure. Proc. Natl. Acad. Sci. U. S. A. 88:8252-8256.

21. Ciurea, A., et al. 2000. Viral persistence in vivo through selection of neutralizing Ab-escape variants. Proc. Natl. Acad. Sci. U. S. A. 97:2749-2754.

22. Wright, K.E., Salvato, M.S., and Buchmeier, M.J. 1989. Neutralizing epitopes of lymphocytic choriomeningitis virus are conformational and require both glycosylation and disulfide bonds for expression. Virology. 171:417-426.

23. Kalinke, U., et al. 1996. The role of somatic mutation in the generation of the protective humoral immune response against vesicular stomatitis virus. Immunity. 5:639-652.

24. Bachmann, M.F., and Zinkernagel, R.M. 1997. Neutralizing antiviral B cell responses. Annu. Rev. Immunol. 15:235-270.

25. Cohen, J. 2003. Public health. AIDS vaccine trial produces disappointment and confusion. Science. 299:1290-1291.

26. Rose, N.F., Roberts, A., Buonocore, L., and Rose, J.K. 2000. Glycoprotein exchange vectors based on vesicular stomatitis virus allow effective boosting and generation of neutralizing Ab's to a primary isolate of human immunodeficiency virus type 1 . J. Virol. 74:10903-10910.

27. Rose, N.F., et al. 2001. An effective AIDS vaccine based on live attenuated vesicular stomatitis virus recombinants. Cell. 106:539-549.

28. Murphy, F.A., Webb, P.A., Johnson, K.M., Whitfield, S.G., and Chappell, W.A. 1970. Arenoviruses in Vero cells: ultrastructural studies. J. Virol. 6:507-518.

29. Buchmeier, M.J. 2002. Arenaviruses: protein structure and function [review]. Curr. Top. Microbiol. Immunol. 262:159-173.

30. Dintzis, H.M., Dintzis, R.Z., and Vogelstein, B. 1976. Molecular determinants of immunogenicity: the immunon model of immune response. Proc. Natl. Acad. Sci. U. S. A. 73:3671-3675.

31. Ochsenbein, A.F., et al. 2000. Correlation of T cell independence of $\mathrm{Ab}$ responses with antigen dose reaching secondary lymphoid organs: implications for splenectomized patients and vaccine design. J. Immunol. 164:6296-6302.

32. Dutko, F.J., and Oldstone, M.B. 1983. Genomic and biological variation among commonly used lymphocytic choriomeningitis virus strains. J. Gen. Virol. 64:1689-1698.

33. Robison, C.S., and Whitt, M.A. 2000. The membrane-proximal stem region of vesicular stomatitis virus $\mathrm{G}$ protein confers efficient virus assembly. J. Virol. 74:2239-2246.

34. Grande-Perez, A., Sierra, S., Castro, M.G., Domingo, E., and Lowenstein, P.R. 2002. Molecular indetermination in the transition to error catastrophe: systematic elimination of lymphocytic choriomeningitis virus through mutagenesis does not correlate linearly with large increases in mutant spectrum complexity. Proc. Natl. Acad. Sci. U. S. A. 99:12938-12943.

35. Bachmann, M.F., Hengartner, H., and Zinkernagel, R.M. 1994. Immunization with recombinant protein: conditions for cytotoxic $\mathrm{T}$ cell and/or Ab induction. Med. Microbiol. Immunol. (Berl.). 183:315-324.

36. Bonilla, W.V., et al. 2002. Effects of promyelocytic leukemia protein on virus-host balance. J. Virol. 76:3810-3818.

37. Battegay, M. 1993. [Quantification of lymphocytic choriomeningitis virus with an immunological focus assay in 24 well plates]. Altex. 10:6-14.

38. Cobbold, S.P., Jayasuriya, A., Nash, A., Prospero, T.D., and Waldmann, H. 1984. Therapy with monoclonal Ab's by elimination of T-cell subsets in vivo. Nature. 312:548-551.

39. Battegay, M., et al. 1993. Impairment and delay of neutralizing antiviral $\mathrm{Ab}$ responses by virus-specific cytotoxic T cells. J. Immunol. 151:5408-5415.

40. Charan, S., and Zinkernagel, R.M. 1986. Ab mediated suppression of secondary IgM response in nude mice against vesicular stomatitis virus. J. Immunol. 136:3057-3061.

41. Scott, D.W., and Gershon, R.K. 1970. Determination of total and merecaptothanol-resistant $\mathrm{Ab}$ in the same serum sample. Clin. Exp. Immunol. 6:313-316.

42. Seiler, P., et al. 1998. Induction of protective cytotoxic $\mathrm{T}$ cell responses in the presence of high titers of virus-neutralizing Ab's: implications for passive and active immunization. J. Exp. Med. 187:649-654.

43. Perez, M., Craven, R.C., and De La Torre, J.C. 2003. The small RING finger protein $Z$ drives arenavirus budding: implications for antiviral strategies. Proc. Natl. Acad. Sci. U. S. A. 100:12978-12983. 